Numerical Study Using CFD of the Cooling Process with Heat Exchangers in Computer Systems

\title{
Estudio Numérico Mediante CFD del Proceso de Enfriamiento con Intercambiadores de Calor en Sistemas Computacionales
}

\author{
$\begin{array}{llll}\text { F. Toapanta } & \text { J. Cortez } & \text { W. Orellana } & \text { W. Quitiaquez } \\ & 1\end{array}$ \\ ${ }^{1}$ Universidad Politécnica Salesiana, Quito, Ecuador \\ E-mail:ltoapanta@ups.edu.ec,jcortezc2@est.ups.edu.ec, \\ worellanac@est.ups.edu.ec,wquitiaquez@ups.edu.ec
}

\begin{abstract}
This scientific article deals with the study and simulation of a radiator that is based on a cross-flow tubular heat exchanger, which has the purpose of cooling the processor, graphics card of a CPU or various hardware in computer systems. Various simulations are carried out in the ANSYS program having various inlet temperatures ranging from $75{ }^{\circ} \mathrm{C}$ to $90{ }^{\circ} \mathrm{C}$ and with different mass flows. The results show that, as the inlet temperature of the fluid to be cooled increases, the outlet of this fluid also increases. However, when mass flow is increased there is a decrease in heat rejection in computational devices.
\end{abstract}

Index terms-- Cooling, CFD, ANSYS, heat exchanger.

\section{Resumen}

El presente artículo científico trata el estudio y simulación de un radiador que se basa en un intercambiador de calor tubular de flujo cruzado, el cual tiene un propósito de refrigerar el procesador, tarjeta gráfica de una CPU o diversos hardware en los sistemas de computación. Se realizan diversas simulaciones en el programa ANSYS teniendo varias temperaturas de ingreso que van en rangos desde 75 ${ }^{\circ} \mathrm{C}$ hasta $90{ }^{\circ} \mathrm{C}$ y con flujos másicos diferentes. Los resultados muestran que, al aumentar la temperatura de ingreso del fluido a refrigerar, la salida de este fluido tambien aumenta. Sin embargo, cuando se aumenta el flujo másico existe una merma en el rechazo de calor en los dispositivos computacionales.

Palabras clave- Enfriamiento, CFD, ANSYS, intercambiador de calor. 


\section{INTRODUCCIÓN}

Tradicionalmente, la mayoría de los centros de procesamiento de datos adoptan refrigeración por aire donde las unidades de aire acondicionado de la sala de computadoras bombean volúmenes de aire refrigerado para enfriar los equipos y eliminan el calor al ambiente. A medida que los centros de datos aumentan de tamaño y los sistemas actuales de refrigeración por aire alcanzan sus limitaciones, la tecnología de refrigeración evolucionará inevitablemente hacia sistemas de refrigeración por líquido-aire (híbrido) o líquido [1], [2], [3]. El enfriamiento por agua de los servidores está emergiendo a las plataformas de computación de alto rendimiento debido a los requisitos para el enfriamiento efectivo de servidores de alta densidad y también para el reciclaje efectivo del calor residual [4].

Los llamados componentes activos, como las CPU y las GPU generan flujos de calor hacia el medio ambiente en promedio de $120 \mathrm{~kW} / \mathrm{m}^{2}$, se pueden enfriar con refrigeración líquida, mientras que los componentes pasivos, como las RAM y los discos duros que generalmente no generan mucho calor, se pueden enfriar con refrigeración por aire, esto propone diseñar un sistema de intercambiador de calor que consiste en disipar el calor de dos fluidos diferentes en un solo sistema, para abordar este concepto, un novedoso intercambiador de calor de triple fluido está diseñado para la refrigeración híbrida en centros de datos [5].

Devdatta y Kulkarni [6] realizaron el diseño y pruebas de un intercambiador de calor de refrigeración líquida monofásica con un poder de disipación de calor de $170 \mathrm{~W}$. En los experimentos se utilizó propilenglicol, para investigar la caracterización del rendimiento térmico del intercambiador de calor. Se encontró que la resistencia térmica cae significativamente para el líquido monofásico, en función del flujo de aire a través del intercambiador de calor. El beneficio de la utilización de este intercambiador de calor es el aumento de la capacidad de potencia de la CPU ya que se incrementa entre un $26 \%$ y un $47 \%$.

En Singapur en el Instituto de Microelectrónica, A*STAR (Agencia de Ciencia, Tecnología e Investigación, por sus siglas en inglés), se desarrolló el diseño de un intercambiador de calor de flujo cruzado que fuera capaz de disipar un calor de $500 \mathrm{~W}$ provenientes de los servidores. El intercambiador de calor de flujo cruzado trabaja con aire y agua como fluidos, además, tiene que ser fabricado con aluminio debido que cumple con los requisitos de rendimiento térmico del sistema de enfriamiento además de su peso ligero [7].

Fan et al. [8] realizaron el diseño analítico de un intercambiador de calor que debe tener un poder de disipación de $215 \mathrm{~W}$ necesario para CPU's de alto TDP (Thermal Design Power, máxima potencia que es capaz de usar un dispositivo). El intercambiador de calor muestra un rendimiento térmico de $0.106^{\circ} \mathrm{C} / \mathrm{W}$ y 0.114 ${ }^{\circ} \mathrm{C} / \mathrm{W}$ para una CPU Xeon Phi de $215 \mathrm{~W}$, con una variación del $5 \%$ en comparación con los resultados de las pruebas térmicas realizadas en el disipador de aire, lo que indica la viabilidad del diseño.

Shedd y Morell [9] encontraron que, el diseño de un sistema de enfriamiento líquido capaz de disipar 1500 $\mathrm{W}$ de calor provenientes de 10 procesadores, conformado por 2 intercambiadores de calor de flujo cruzado y 10 "bloques de CPU, es más eficiente que un sistema tradicional de refrigeración por viento, mostrando un una mejora del $55 \%$ en el procesamiento computacional, reducción del ruido producido por el sistema de refrigeración y finalmente el sistema mantiene una temperatura constante e igual en los 10 procesadores con un margen de error de $+/-2{ }^{\circ} \mathrm{C}$

En Electronic Cooling Solutions Inc. se realizó el diseño y construcción de un sistema de refrigeración líquida capaz de enfriar un chip procesador de alta densidad de potencia, el diseño consiste en un intercambiador de calor de flujo cruzado y demostró que con un flujo de refrigerante (Novec 649 de la marca 3 m) de 1.39 litros/min tiene una capacidad de disipación de $1400 \mathrm{~W}$ de antes de alcanzar el límite de bloque de cobre de $100{ }^{\circ} \mathrm{C}$ (temperatura máxima de un procesador). Este diseño demostró ser más eficiente que el diseño tradicional de refrigeración por viento y diseños alternativos de refrigeración líquida [10].

Se encontró en un estudió de Kheirabadi y Groulx [11] que, para la electrónica del servidor se ha presentado soluciones de enfriamiento líquido, las cuales han exhibido mejoras significativas en términos de las limitaciones de la transferencia de calor, temperaturas del refrigerante y caudales de refrigerante en relación con enfriamiento de aire tradicional.

Las temperaturas promedio más altas de CPU y placa madre (MB) del sistema de enfriamiento del disipador de calor indican que, no es un sistema de enfriamiento efectivo en comparación con los sistemas de refrigeración por agua y refrigeración termoeléctrica, los valores de rendimiento son inversamente proporcionales a la temperatura promedio de la CPU y, por lo tanto, el sistema de enfriamiento termoeléctrico, que tiene la CPU con la temperatura promedio más baja, ofrece el mayor rendimiento de la CPU [12].

Una solución de enfriamiento recomendada es un sistema de enfriamiento líquido de contacto térmico. Este sistema elimina los riesgos de fugas presentes en los sistemas convencionales de refrigeración por agua mediante la sustitución de sus conectores fluídicos con un intercambiador de calor de contacto térmico, el inconveniente adicional fue el calor suplementario de las interfaces de transferencia que sirvieron para aumentar la temperatura general y la resistencia del sistema [13]. 
Un sistema de enfriamiento tradicional comparado con un sistema de doble capa planteado en Wang, et al. [14], muestra que el conjunto otorga un rendimiento de enfriamiento mejorado en $4.11 \%, 0.98 \%$ y $0.85 \mathrm{de} 0 \mathrm{~m} / \mathrm{s}$ a $8 \mathrm{~m} / \mathrm{s}$, no obstante, deteriorado en $0.48 \%$ y $0.52 \%$ con viento fuertes con velocidades de $12 \mathrm{~m} / \mathrm{s}$ y $16 \mathrm{~m} / \mathrm{s}$. Este modelo propuesto podría recomendarse para alta capacidad de plantas de energía, en lugares áridos con pequeñas velocidades del viento durante todo el año.

La orientación óptima para el sistema de enfriamiento permite extraer la máxima disipación de energía térmica es la posición del termosifón, que permite eliminar entradas de calor de hasta $150 \mathrm{~W}$ si se tiene en cuenta que la CPU funciona de forma segura con una temperatura de unión continua inferior a $90{ }^{\circ} \mathrm{C}$. Para estas mismas condiciones de funcionamiento, en posiciones horizontales y con antigravedad, el sistema de enfriamiento permite eliminar entradas de calor de hasta $100 \mathrm{~W}$ y $50 \mathrm{~W}$, respectivamente [15].

Un sistema Propuesto en Sun et al. [16] consta de una unidad de refrigeración por compresión de vapor conectada con una unidad de tubería de calor separada por un condensador evaporativo, en comparación con la unidad de expansión directa de enfriamiento por aire y el enfriamiento por aire enfriador de doble fuente, el sistema propuesto tiene el menor consumo anual de energía con la mayor energía eficiencia. Por lo que es la opción preferida para centros de datos en funcionamiento durante todo el año, especialmente en regiones con baja temperatura media anual.

Con el fin de proponer otras opciones a un intercambiador de calor, en el modelo matemático de Liang et al. [17], para tubo de calor y el sistema termoeléctrico, en términos de entrada de energía eléctrica y capacidad de enfriamiento a varias temperaturas, el HP (Heat Pipe) mejoró su capacidad y el coeficiente de rendimiento (COP) del sistema TE (Thermoelectric) en un $53 \%$ y $42 \%$ respectivamente a una temperatura final fría de $10^{\circ} \mathrm{C}$. La tubería de calor es más conveniente para enfriar dispositivos grandes a temperaturas más altas.

En el presente artículo se pretende realizar el diseño de un intercambiador de calor para el enfriamiento de procesadores AMD Epyc, reemplazando el sistema de refrigeración por viento a un sistema de refrigeración líquida, mediante el intercambiador de flujo cruzado, y dicho diseño será comprobado con el estudio numérico de donde se la selección del mejor material para la construcción del intercambiador de calor.

\section{MATERIALES Y MÉTODOS}

El presente documento trata sobre el diseño y simulación de un sistema de enfriamiento líquido, en el programa computacional ANSYS para determinar el comportamiento del mismo, el sistema consta de diferentes elementos como lo muestra la Fig. 1, cuenta con un depósito, en el cual se encuentra alojado el líquido, una bomba que se encarga de extraer el fluido para llevarlo hacia el bloque, saliendo del mismo el fluido caliente, ingresa al radiador, elemento del cual se realizara el análisis, y finalmente en este volver a enfriar el fluido logrando así que se repita el ciclo.

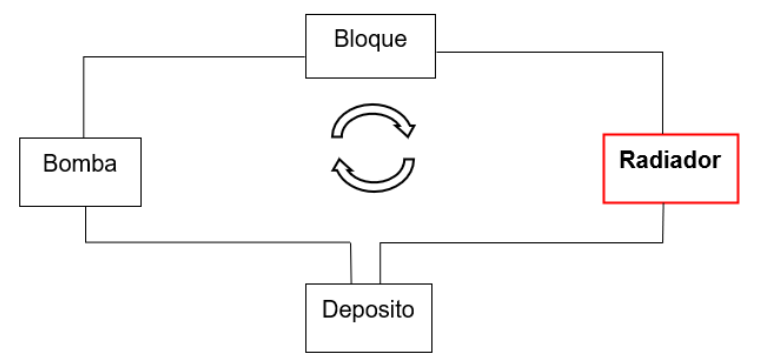

Figura 1: Diagrama de funcionamiento del sistema de enfriamiento

El llamado "Bloque" del sistema tiene un objetivo claro, el cual es, enrutar el líquido refrigerante hacia el lugar que el usuario requiere enfriar de una manera más eficiente. Su apariencia física suele estar formada por alteas o micro aletas, de tal manera que el fluido pasa entre ellas y absorbe el calor generado para posteriormente seguir con el proceso.

El diseño del radiador tiene que ser capaz de disipar más calor del que absorbe y en el escenario más extremo posible, disipar el mismo calor que recolecta de los servidores, para lograr este objetivo hay que tener en cuenta varios factores como lo son, el aire ambiente, los ventiladores, entre otros.

\subsection{Funcionamiento del Radiador}

El funcionamiento del radiador comprende varios pasos, el líquido entra caliente por un agujero y sale por el otro extremo a menor temperatura. Mientras viaja por dentro del radiador recorre varias zonas sensibles a la temperatura ambiente que se encuentran más frías. Al pasar por esta zona fresca, el líquido disipa calor antes de retornar al depósito.

Para disipar calor el radiador cuenta con aletas de metal muy finas unidas a los tubos por donde pasa el líquido, estas aletas en conjunto con los ventiladores y el aire exterior, enfrían el tubo del radiador y a su vez la del líquido que pasa por su interior.

Los radiadores generalmente se los encuentra de dos tipos de materiales, de aluminio y de cobre, estos, con respecto a los tubos y sistemas de disipación de calor ya que las otras partes que conforman el radiador como son los racores donde se conectan las mangueras y las partes laterales son de latón simplemente.

El cobre y el aluminio como se conoce son materiales que se calientan y se enfrían con mucha facilidad y rapidez. El radiador diseñado y analizado en 
el presente documento se lo realizará de cobre y latón, esto debido a las características favorables entre uno y otro, ya que, al introducir aluminio en algún componente de este, se produciría reacciones desfavorables entre los materiales como lo es la corrosión galvánica.

El radiador que es instalado en una determinada parte de la CPU cuenta con una entrada y salida del agua destilada, el enfriamiento se llevará a cabo con la ayuda del flujo de aire como se muestra en la Fig. 2, cabe recalcar que aire provendrá de ventiladores.

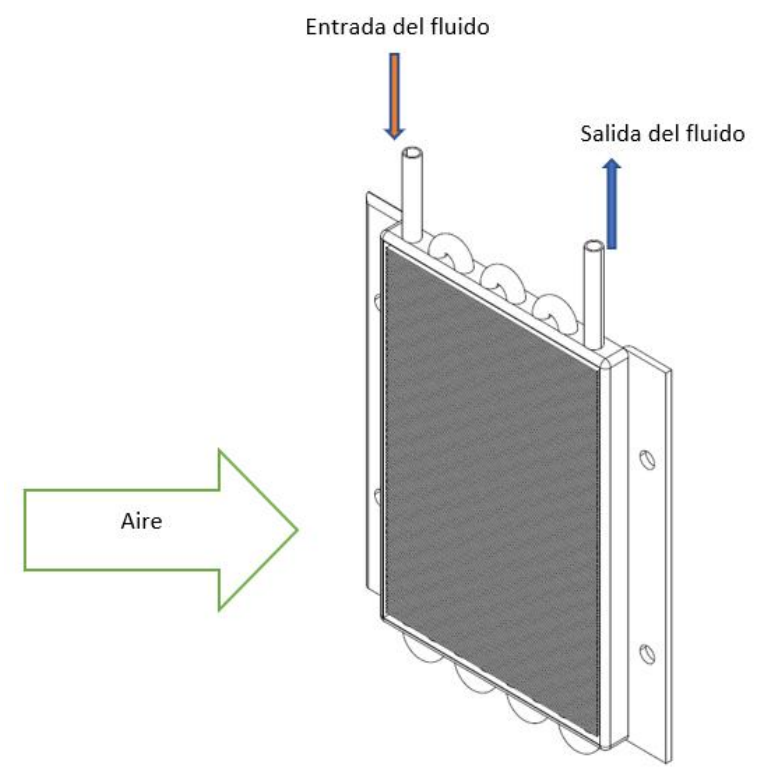

Figura 2: Esquema de funcionamiento del disipador

Las dimensiones principales del radiador para enfriamiento de procesadores son: $11.5 \mathrm{~cm}$ de largo y $7.9 \mathrm{~cm}$ de ancho, en la Fig. 3 se aprecian estas dimensiones, además, de otras longitudes fundamentales para este estudio numérico.

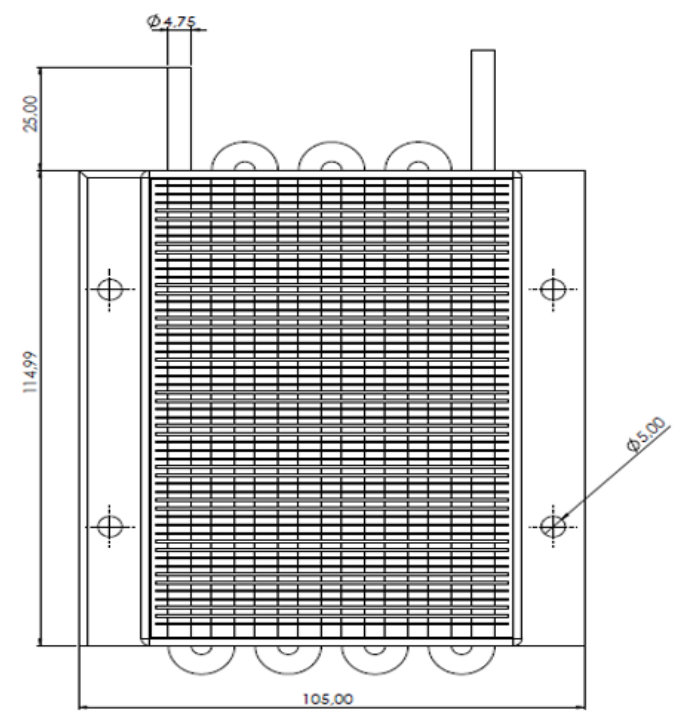

Figura 3: Dimensiones del radiador
Para el análisis teórico del calor disipado, así como las temperaturas de entrada, salida y del procesador o bien tarjeta gráfica que se utilice como elemento a enfriar se utilizan valores de flujo másico de agua y aire de equipos comerciales tentativos para la construcción de este modelo de intercambiador de calor los parámetros principales se presentan en la tabla 1. Por otro lado, el disipador es un intercambiador de calor de tubos extendidos, fabricado de cobre y se realiza el análisis de este dispositivo por su versatilidad de aplicación y construcción.

Tabla 1: Parámetros principales de análisis

\begin{tabular}{ccc}
\hline Parámetro & Agua & Aire \\
\hline \hline Flujo másico, $[\mathrm{kg} / \mathrm{s}]$ & 0,14 & 9 \\
$\begin{array}{c}\text { Calor específico, } \\
{[\mathrm{J} / \mathrm{kgK}]}\end{array}$ & 4190 & 1005 \\
$\begin{array}{c}\text { Temperatura de } \\
\text { entrada }(\mathrm{T} \text { in }),\left[{ }^{\circ} \mathrm{C}\right]\end{array}$ & $75-86$ & $20-30$ \\
\hline
\end{tabular}

La temperatura de entrada del aire se considera un valor de entre 20 y $30{ }^{\circ} \mathrm{C}$ adoptando los valores menores si el intercambiador de calor se encuentra en Quito y valores máximos en lugares más calurosos como en la región Costa.

Se asume el rango de 75 a $86{ }^{\circ} \mathrm{C}$ al observar estadísticas y experiencias personales de procesadores y tarjetas gráficas utilizadas en un día normal, llegando a una temperatura máxima y recomendable de $86^{\circ} \mathrm{C}$.

$\mathrm{Al}$ determinar las diferentes variables de interés que se representan posteriormente en la simulación se utiliza el método de NTU (número de unidades de transferencia). Los resultados obtenidos en base a temperatura ambiente de Quito y temperatura máxima del procesador.

\subsection{Modelado Numérico}

En el modelado de un intercambiador de calor de flujo cruzado, el modelo de simulación que se elige es K-épsilon RNG $(\mathrm{k}-\varepsilon)$, debido a que este modelo de turbulencia contempla superficies curvas presentes en el intercambiador de calor.

El modelo de turbulencia basado en RNG $(\mathrm{k}-\varepsilon)$ se deriva de las ecuaciones instantáneas de Navier-Stokes, utilizando una técnica matemática llamada métodos de "grupo de renormalización" (RNG).

Al ser un intercambiador diseñado con curvas, es necesario la aplicación de este método kappa épsilon RNG, en la ecuación 1 presentada a continuación representa el modelo de turbulencia para la energía cinética kappa.

El modelo de turbulencia basado en RNG $(\mathrm{k}-\varepsilon)$ se deriva de las ecuaciones instantáneas de Navier-Stokes, 
utilizando una técnica matemática llamada métodos de "grupo de renormalización" (RNG).

Al ser un intercambiador diseñado con curvas, es necesario la aplicación de este método kappa épsilon RNG, en la ecuación 1 presentada a continuación representa el modelo de turbulencia para la energía cinética kappa.

$$
\frac{\partial}{\partial t}(\rho \kappa)+\frac{\partial}{\partial x_{i}}\left(\rho \kappa \mu_{i}\right)=\frac{\partial}{\partial x_{j}} \mu_{e f f}\left(\frac{\partial \kappa}{\partial x_{j}}\right)+G_{\kappa}+G_{b}-\rho \varepsilon-Y_{M}+S_{\kappa}
$$

La ecuación 2 representa la difusividad épsilon en el modelo de turbulencia.

$$
\begin{aligned}
& \frac{\partial}{\partial t}(\rho \varepsilon)+\frac{\partial}{\partial x_{i}}\left(\rho \varepsilon \mu_{i}\right)= \\
& \frac{\partial}{\partial x_{j}} \mu_{e f f} \alpha_{\varepsilon}\left(\frac{\partial \varepsilon}{\partial x_{j}}\right)+C_{1 \varepsilon}\left(\frac{\varepsilon}{\kappa}\right)\left(G_{\kappa}+C_{3 \varepsilon} G_{b}\right)-C_{2 \varepsilon} \rho\left(\frac{\varepsilon^{2}}{\kappa}\right)-R_{\varepsilon}+S_{\varepsilon}
\end{aligned}
$$

El procedimiento de eliminación de incrustaciones en la teoría RNG da como resultado una ecuación diferencial para la viscosidad turbulenta como se muestra en la ecuación 3.

$$
d\left(\frac{\rho^{2} \kappa}{\sqrt{\varepsilon \mu}}\right)=1.72 \frac{\vec{v}}{\sqrt{\vec{v}-1+C_{v}}} d \vec{v}
$$

La ecuación para conservación de la masa o ecuación de continuidad y ecuación de conservación de momento pueden ser escritas de la siguiente forma:

$$
\begin{gathered}
\frac{\partial \rho}{\partial t}+\nabla \cdot(\rho \vec{v})=S_{m} \\
\frac{\partial}{\partial t}(\rho \vec{v})+\nabla \cdot(\rho \vec{v} \vec{v})=-\nabla p+\rho \vec{g}+\vec{F}
\end{gathered}
$$

Donde:

$$
\nabla \cdot \vec{v}=\frac{\partial v_{x}}{\partial x}+\frac{\partial v_{r}}{\partial r}+\frac{v_{r}}{r}
$$

Por otro lado, la ecuación para la conservación de la energía esta descrita de la siguiente forma:

$$
\frac{\partial}{\partial t}(\rho E)+\nabla \cdot(\vec{v}(\rho E+p))=-\nabla \cdot\left(\sum_{j} h_{j} J_{j}\right)+S_{h}
$$

\subsection{Mallado}

Mediante el análisis se pretende obtener los datos de temperatura de salida de agua fría del intercambiador de flujo cruzado diseñado para el presente artículo científico. El objeto principal para analizar en las simulaciones se va a centrar en el tubo del intercambiador de calor.

La malla que se muestra en la Fig. 4 se obtuvo después de validar la operación de mallado con la herramienta "Body Sizing" considerando un tamaño de elemento igual a $0,75 \mathrm{~mm}$, no se consideró un menor tamaño para mantener la malla con un número menor a 500000 elementos, esta estrategia de mallado fue aplicada tanto a la parte del tubo como a la parte del líquido en su interior.

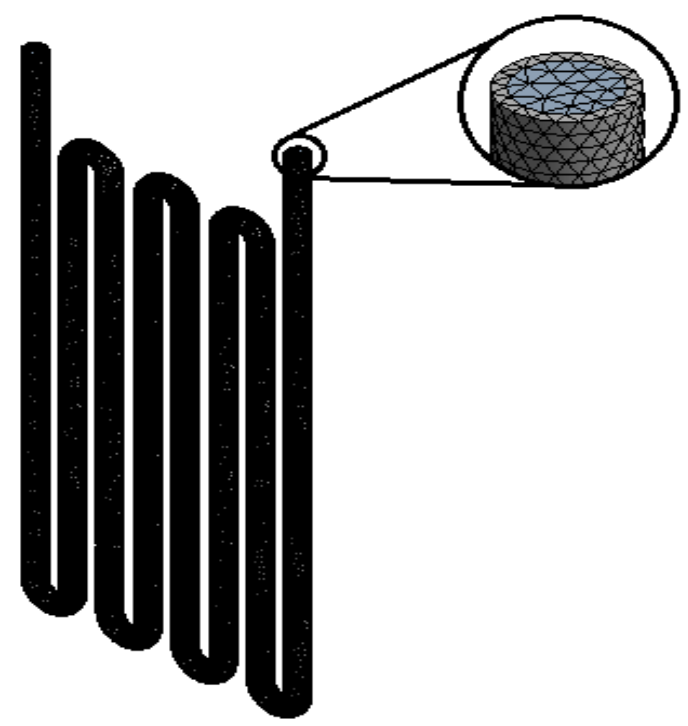

Figura 4: Mallado del intercambiador de calor con detalle

Es importante comprobar la convergencia del mallado, en la Fig. 5 se observa una convergencia de malla bajo el enfoque de ortogonalidad, dando un valor numérico promedio de 0,7862 . Este es adecuado para proceder con las simulaciones previstas.

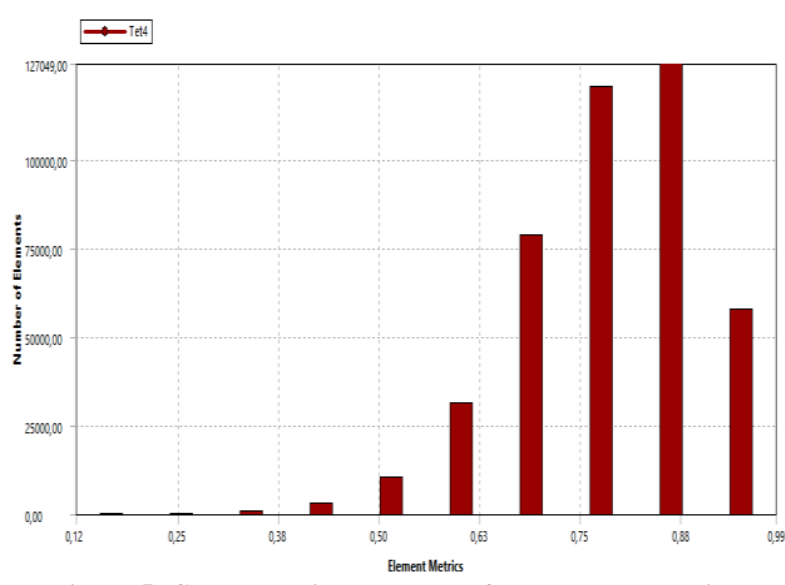

Figura 5: Convergencia de malla, enfoque de ortogonalidad 
Por otro lado, se corrobora la calidad de mallado con el enfoque de oblicuidad o Skewness para el mallado de análisis, en la Fig. 6 se presenta el promedio de oblicuidad, obteniendo un valor numérico promedio de 0.221 , el mismo que se encuentra en el rango de mallado excelente.

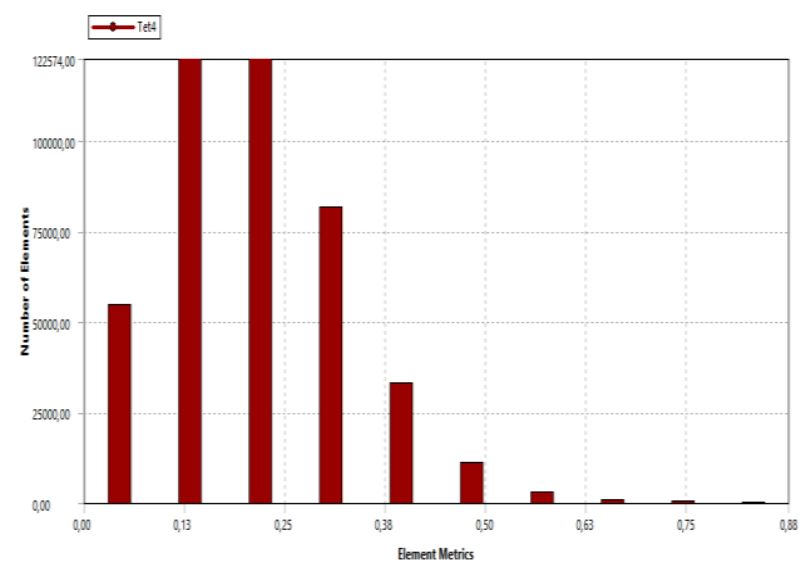

Figura 6: Convergencia de malla, enfoque de oblicuidad

\section{RESULTADOS Y DISCUSIÓN}

Con el objetivo de obtener una variedad de resultados, condiciones estándar tomando en cuenta la temperatura ambiente en donde se encuentre el equipo, más específicamente la computadora que este equipado el sistema de enfriamiento y los valores de flujo másicos presentado en la tabla 1 de la sección anterior se presentan simulaciones con valores de temperaturas máximas y mínimas, así como variación del flujo de agua y calor disipado del procesador.

Todas las simulaciones inician bajo el modelo de turbulencia $\mathrm{k}-\varepsilon$ RNG debido a que este es el mejor método para analizar con precisión los cambios ocurridos en las secciones curvas del tubo en forma de serpentín.

Se inicia las simulaciones con una temperatura de entrada del agua de $86{ }^{\circ} \mathrm{C}$ que, se traduce a la temperatura del procesador máxima recomendable según fabricantes para el trabajo exigente de un computador. Se obtuvieron resultados de temperatura de salida de $20.267{ }^{\circ} \mathrm{C}$ frente a una temperatura esperada de $20{ }^{\circ} \mathrm{C}$ según los cálculos teóricos se observa un rendimiento eficaz, que otorga una viabilidad aprobada en la construcción de este sistema. Con esto se realiza la comprobación del programa, métodos y modelos, utilizados para este estudio numérico.

La Fig. 7, corresponde al enfriamiento del agua que se utiliza para refrigerar las unidades computacionales, se observa un ingreso de temperatura $85.3{ }^{\circ} \mathrm{C}$ y una salida de $22.31^{\circ} \mathrm{C}$, se obtiene esta temperatura de salida al hacer circular el agua con un flujo másico de 0.14 $\mathrm{kg} / \mathrm{s}$.

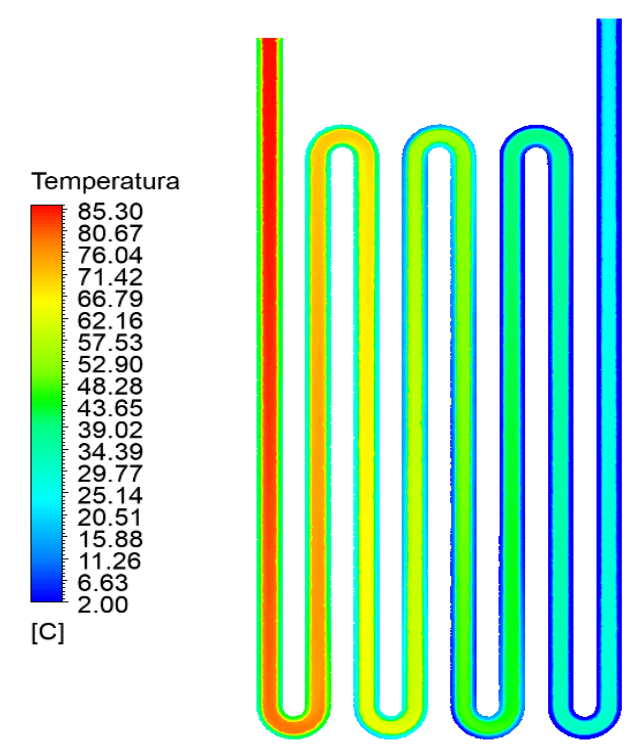

Figura 7: Intercambiador de calor, entrada y salida de temperaturas

Como complemento, en la Fig. 8, se observa un contorno que muestra las variaciones de turbulencia que tiene el agua dentro del tubo en forma de serpentín. Se aprecia que las partes de mayor turbulencia están ubicadas en las curvaturas del tubo, una vez superada esa sección, la turbulencia decrece dramáticamente.

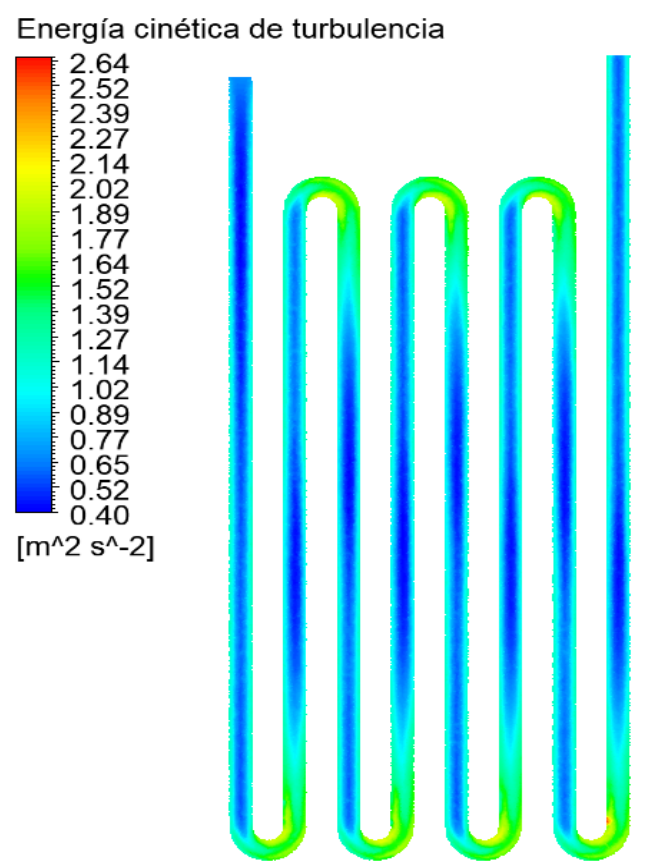

Figura 8: Energía cinética de turbulencia en el serpentín

En otra simulación con una temperatura del procesador de $90{ }^{\circ} \mathrm{C}$, que presenta un caso extremo, se toma esta temperatura como un caso de suposición, debido a que los ventiladores pueden estar en mal estado o existir otros daños en el hardware, los resultados obtenidos de temperatura de salida del agua 
de $26{ }^{\circ} \mathrm{C}$, en este caso el intercambiador de calor resuelve el problema de la alta temperatura.

En la Fig. 9, se visualiza el descenso de temperatura para las dos simulaciones anteriores, la primera con temperatura de ingreso de agua de $86{ }^{\circ} \mathrm{C}$ y la segunda para la temperatura de ingreso de $90{ }^{\circ} \mathrm{C}$, estas analizadas en el último tubo del serpentín de refrigeración. Se logra que la temperatura de salida llegue hasta $26{ }^{\circ} \mathrm{C}$, esta se encuentra en un rango aceptable de enfriamiento del agua.

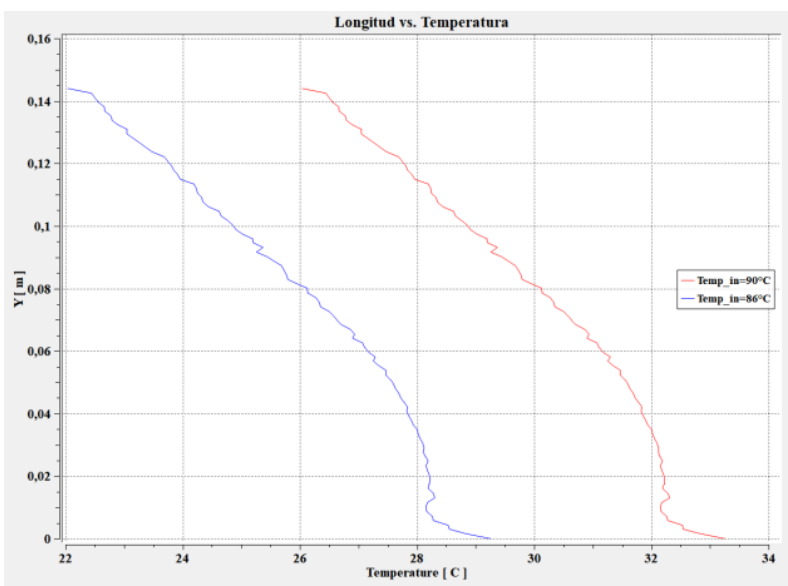

Figura 9: Comparación de la temperatura de salida con diferentes temperaturas de ingreso

Por otro lado, en la Fig. 10, se observa la variación de la temperatura de salida cuando la capacidad calórica que se desprende del intercambiador aumenta, desde $38.7 \mathrm{~kW}$, que es la capacidad calórica de diseño, hasta $39 \mathrm{~kW}$, estas con temperatura de ingreso de $90{ }^{\circ} \mathrm{C}$. La temperatura de salida, para la capacidad calórica de diseño, se obtiene de $26{ }^{\circ} \mathrm{C}$, mientras que, para la variación de la capacidad calórica de salida la temperatura obtenida es de $23.9{ }^{\circ} \mathrm{C}$. Esto se traduce a que, si se aumenta la salida de calor la temperatura de refrigeración disminuye.

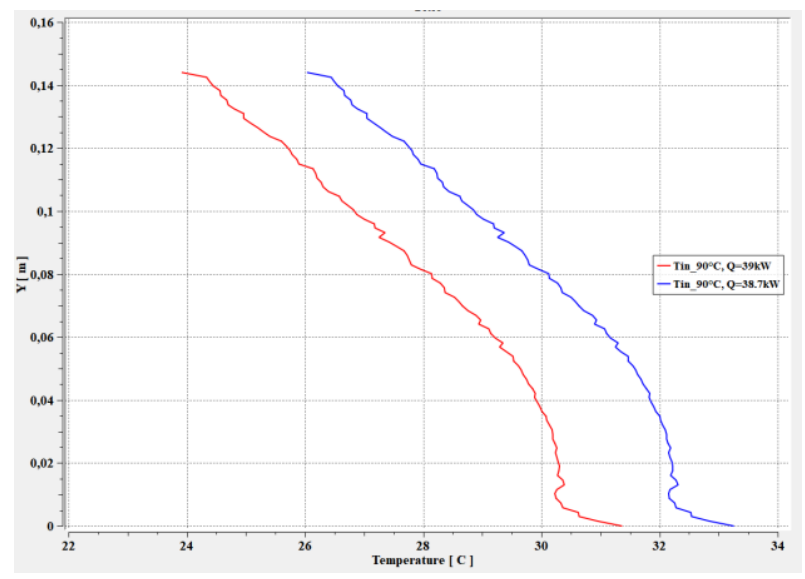

Figura 10: Comparación de la temperatura de salida con diferentes capacidades de calor

Se realiza otra simulación, manteniendo la temperatura del procesador de $75{ }^{\circ} \mathrm{C}$ que viene a ser un caso de trabajo normal entre moderado y pesado y con un aumento del flujo másico del fluido de $0,17 \mathrm{~kg} / \mathrm{s}$ que se debería al cambio de la bomba del sistema, se observa la temperatura de salida de $22.7^{\circ} \mathrm{C}$. Esto se aprecia en la Fig. 11.

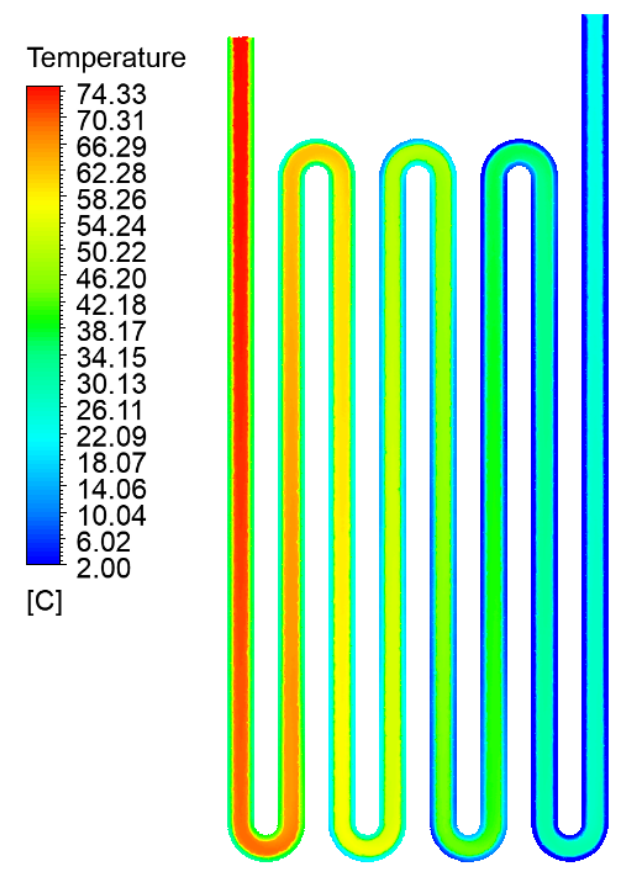

Figura 11: Contorno de temperatura con $T=7^{\circ} \mathrm{C}$

Finalmente, la Fig. 12 muestra el cambio de temperatura en todo el intercambiador, al inicio se tiene una temperatura de $75{ }^{\circ} \mathrm{C}$ y a la salida se observa una temperatura de $20^{\circ} \mathrm{C}$.

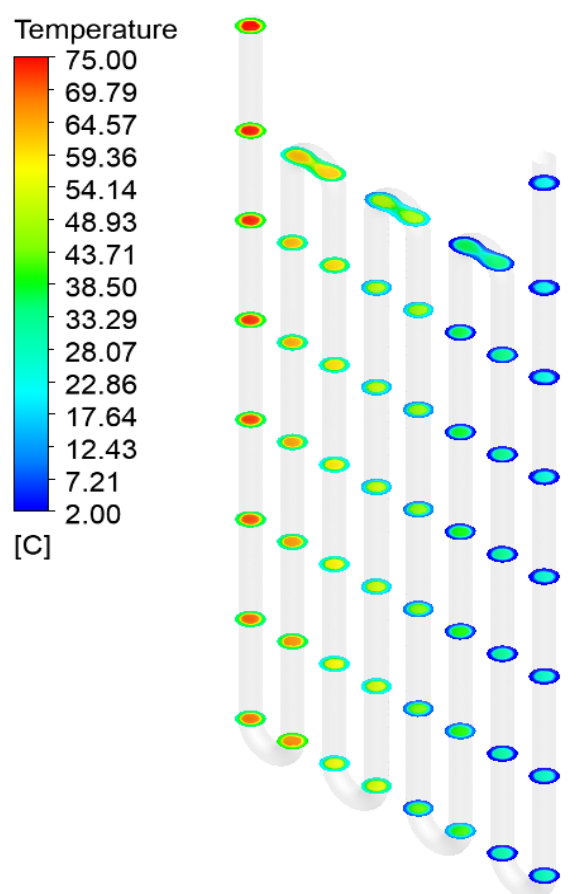

Figura 12: Cambio de temperatura en el intercambiador 
La temperaturas alcanzadas en las diversas simulaciones realizadas se traducen en un acierto con respecto al análisis teórico con lo cual el intercambiador de calor propuesto es idóneo para el trabajo del presente artículo, la construcción del proyecto sería una opción a los sistemas de refrigeración comerciales al obtener unos resultados prácticamente eficientes y que sirven al usuario, al comparar las temperaturas obtenidas con los resultados del cálculo previo, podemos determinar que tan solo se presenta un error porcentual de un $1.34 \%$, se observa que el comportamiento del intercambiador de calor es bueno. A su vez como trabajo complementario se realizaron diversos casos de simulación abarcando cualquier situación que se presente en un día ordinario con el computador obteniendo resultados satisfactorios al encontrar una refrigeración suficiente si el procesador está a su máxima temperatura que es de $90^{\circ} \mathrm{C}$.

En la Fig. 13, se aprecia como la temperatura del agua se va reduciendo a medida que circula por el intercambiador de calor, al principio se observa que el agua ingresa a $55^{\circ} \mathrm{C}$, al tiempo de menos de $1 \mathrm{~s}$ el agua desciende hasta el valor de $22.5^{\circ} \mathrm{C}$, con esto se logra el objetivo de este estudio, constatar la reducción de temperatura del agua que recircula por todo el servidor de datos computacionales.

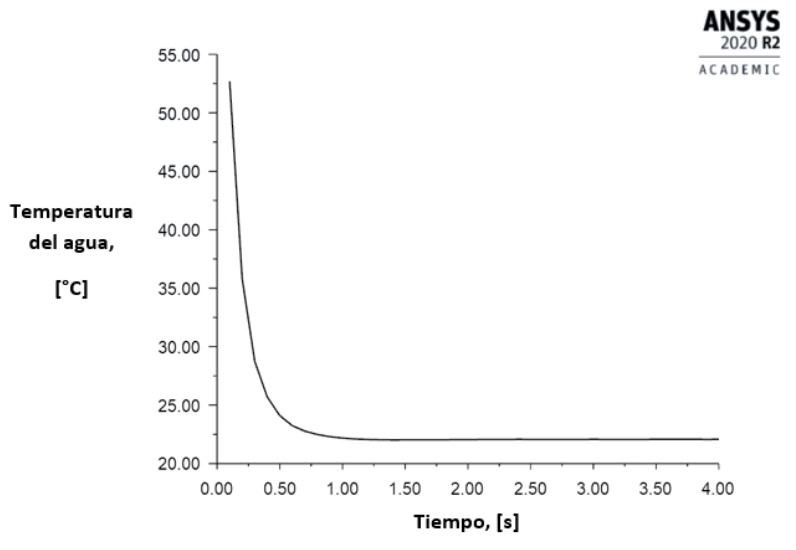

Figura 13: Variación de temperatura del agua en función del tiempo

\section{CONCLUSIONES Y RECOMENDACIONES}

En función a los resultados obtenidos, se pueden plantear las siguientes conclusiones:

- Se realizó la simulación numérica para el tubo del radiador, con diámetro hidráulico de $3.75 \mathrm{~mm}$, diseñado previamente en SOLID WORKS, mediante el software ANSYS CDF. La simulación se elaboró en estado estacionario, utilizando agua como fluido de trabajo, con una temperatura inicial de $86{ }^{\circ} \mathrm{C}$, una salida de calor previamente calculada por la pared externa del tubo de cobre y un flujo másico igual a $0.14 \mathrm{~kg} / \mathrm{s}$.

- La transferencia de calor que ocurre desde el agua, hacia el tubo y posteriormente al ambiente, fue numéricamente simulada con un flujo de agua que atraviesa toda la tubería en forma de serpentín, se utilizó el modelo de turbulencia kappa-épsilon RNG debido que este modelo considera de una manera más precisa la turbulencia ocurrida en las secciones curvas presentes en la tubería.

- Al utilizar agua como fluido para refrigerar tiene como ventaja su gran capacidad de enfriamiento en comparación con el aire, ya que su capacidad calorífica es cuatro veces mayor.

- Al momento de comparar la temperatura de salida calculada por simulación en ANSYS CFD con la temperatura de salida obtenida por cálculos realizados a mano, se nota que existe un error de tan solo $1.34 \%$, esto comprueba la viabilidad de la simulación.

- Cuando se supone una temperatura de entrada del agua igual a $90{ }^{\circ} \mathrm{C}$ (por altas exigencias en el procesador), se observa una temperatura de salida de $24.285{ }^{\circ} \mathrm{C}$, lo cual, si bien es adecuado para mantener el procesador a una temperatura de trabajo, se pueden realizar modificaciones en los ventiladores o la bomba de agua para ajustar la temperatura de salida a $20{ }^{\circ} \mathrm{C}$ que sería lo óptimo.

\section{AGRADECIMIENTOS}

Los autores de esta investigación agradecen a la carrera de ingeniería mecánica de la Universidad Politécnica Salesiana, al Grupo de Investigación en Energías Renovables e Implementación Mecánica de Pymes GIERIMP y al Branch ASHRAE UPS-QUITO, por sus grandes aportes en este estudio.

\section{REFERENCIAS BIBLIOGRÁFICAS}

[1] A. Addagatla, J. Fernandes, D. Mani, D. Agonafer, and V. Mulay, "Effect of warm water cooling for an isolated hybrid liquid cooled server," Annu. IEEE Semicond. Therm. Meas. Manag. Symp., vol. 2015April, pp. 203-207, 2015, doi: 10.1109/SEMITHERM.2015.7100161.

[2] K. Nemati, T. Gao, B. T. Murray, and B. Sammakia, "Experimental characterization of the rear door fans and heat exchanger of a fullyenclosed, hybrid-cooled server cabinet," Annu. IEEE Semicond. Therm. Meas. Manag. Symp., vol. 2015-April, pp. 155-162, 2015, doi: 10.1109/SEMI-THERM.2015.7100154.

[3] M. A. Kadhim, Y. T. Al-Anii, N. Kapur, J. L. Summers, and H. M. Thompson, "Performance of a mixed mode air handling unit for direct liquidcooled servers," Annu. IEEE Semicond. Therm. Meas. Manag. Symp., pp. 172-178, 2017, doi: 10.1109/SEMI-THERM.2017.7896926.

[4] S. J. Ovaska, R. E. Dragseth, and S. A. Hanssen, "Impact of retrofitted CPU water cooling on supercomputer performance and power 
consumption," Conf. Proc. - IEEE SOUTHEASTCON, vol. 2016-July, pp. 1-2, 2016, doi: 10.1109/SECON.2016.7506669.

[5] N. Raja Kuppusamy and L. Poh Seng, "Study on thermal and hydrodynamic performance of a triple fluid heat exchanger with different passes and rows," Energy Procedia, vol. 158, pp. 5901-5906, 2019, doi: 10.1016/j.egypro.2019.01.534.

[6] D. P. Kulkarni and R. Steinbrecher, "Compact liquid enhanced air cooling thermal solution for high power processors in existing air-cooled platforms," Annu. IEEE Semicond. Therm. Meas. Manag. Symp., vol. Part F1214, pp. 81-85, 2016, doi: 10.1109/SEMI-THERM.2016.7458449.

[7] G. Tang, Y. Han, and X. Zhang, Compact heat exchanger design and energy efficiency optimization for data centre cooling application, vol. 2018-Febru. 2018.

[8] Y. Fan, C. Winkel, D. Kulkarni, and W. Tian, "Analytical Design Methodology for Liquid Based Cooling Solution for High TDP CPUs," Proc. 17th Intersoc. Conf. Therm. Thermomechanical Phenom. Electron. Syst. ITherm 2018, pp. 582 586, 2018, doi: 10.1109/ITHERM.2018.8419562.

[9] T. A. Shedd and R. A. Morell, "Cooling 11.6 TFlops (1500 watts) in an office environment," Annu. IEEE Semicond. Therm. Meas. Manag. Symp., pp. 122-124, 2017, doi: 10.1109/SEMITHERM.2017.7896918.

[10]G. R. Wagner et al., "Test results from the comparison of three liquid cooling methods for high-power processors," Proc. 15th Intersoc. Conf. Therm. Thermomechanical Phenom. Electron. Syst. ITherm 2016, pp. 619-624, 2016, doi: 10.1109/ITHERM.2016.7517605.

[11]A. C. Kheirabadi and D. Groulx, "Cooling of server electronics: A design review of existing technology," Appl. Therm. Eng., vol. 105, no. 2016, pp. 622-638, 2016, doi: 10.1016/j.applthermaleng.2016.03.056.

[12] S. O. Tan and H. Demirel, "Performance and cooling efficiency of thermoelectric modules on server central processing unit and Northbridge," Comput. Electr. Eng., vol. 46, pp. 46-55, 2015, doi: 10.1016/j.compeleceng.2015.07.012.

[13]A. C. Kheirabadi and D. Groulx, "Experimental evaluation of a thermal contact liquid cooling system for server electronics," Appl. Therm. Eng., vol. 129, pp. 1010-1025, 2018, doi: 10.1016/j.applthermaleng.2017.10.098.

[14] W. Wang, L. Chen, Y. Kong, L. Yang, Y. Niu, and $\mathrm{X}$. Du, "Cooling performance evaluation for double-layer configuration of air-cooled heat exchanger," Int. J. Heat Mass Transf., vol. 151, p. 119396, 2020, doi: 10.1016/j.ijheatmasstransfer.2020.119396.

[15] S. Maalej, A. Zayoud, I. Abdelaziz, I. Saad, and M. C. Zaghdoudi, "Thermal performance of finned heat pipe system for Central Processing Unit cooling," Energy Convers. Manag., vol. 218, no. February, p. 112977, 2020, doi: 10.1016/j.enconman.2020.112977.

[16] Y. Sun, T. Wang, L. Yang, L. Hu, and X. Zeng, "Research of an integrated cooling system consisted of compression refrigeration and pumpdriven heat pipe for data centers," Energy Build., vol. 187, pp. 16-23, 2019, doi: 10.1016/j.enbuild.2019.01.050.

[17] K. Liang, Z. Li, M. Chen, and H. Jiang, "Comparisons between heat pipe, thermoelectric system, and vapour compression refrigeration system for electronics cooling," Appl. Therm. Eng., vol. 146, no. September 2018, pp. 260-267, 2019, doi: 10.1016/j.applthermaleng.2018.09.120.

[18]L. Bilurbina Alter, F. Liesa Mestres, and J. I. Iribarren Laco, Corrosión y protección, vol. 53, no. 9. 2003.

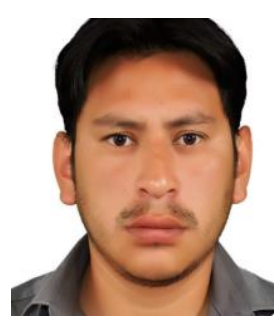

Fernando Toapanta Ramos.Nació en Quito, Ecuador en 1986. Recibió su título de Ingeniero Mecánico de la Universidad Politécnica Salesiana, sede Quito en 2012; de Master en Gestión de energías de la Universidad Técnica de Cotopaxi, Latacunga en 2016; y esta terminando su tesis doctoral en ingeniería, en la Universidad Pontificia Bolivariana, Medellín-Colombia. Sus campos de investigación están relacionados con la mecánica de fluidos, termodinámica, transferencia de calor y simulaciones de procesos en cambio de fase mediante ANSYS.

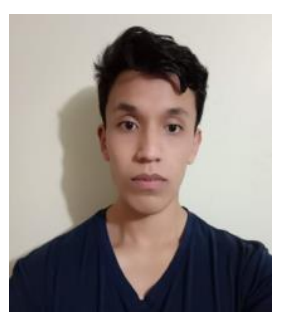

Jairo Cortez Chamorro.- Nació en Quito en 1997. Esta en el último semestre de la carrera de ingeniería Mecánica en la Universidad Politécnica Salesiana, sede Quito. Sus campos de investigación están relacionados con el diseño mecánico, energía térmica, termodinámica y transferencia de calor. 


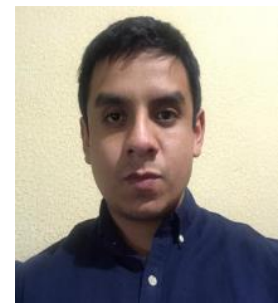

Wilson Orellana Chicaiza. Nació en Quito en 1993. Esta en el último semestre de la carrera de ingeniería Mecánica en la Universidad Politécnica Salesiana, sede Quito. Sus campos de investigación están relacionados con el diseño mecánico, energía térmica y cálculo estructural.

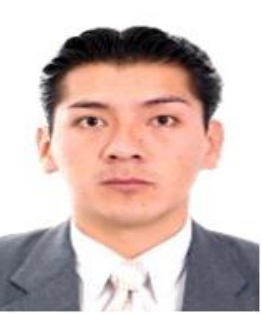

William Quitiaquez. - Nació en Quito en 1988. Recibió su título de Ingeniero Mecánico de la Universidad Politécnica Salesiana en 2011; de Magister en Gestión de Energías de la Universidad Técnica de Cotopaxi, en 2015; de Magister en Ingeniería de la

Universidad Pontificia Bolivariana de Medellín, en 2019. Actualmente, obtuvo la distinción de Candidato a Doctor en la Universidad Pontificia Bolivariana de Medellín, su campo de investigación se encuentra relacionado a Fuentes Renovables de Energía, Termodinámica, Transferencia de Calor y Simulación. 